\title{
Inferior Vena Cava Hypoplasia Associated with Deep Vein Thrombosis - Case Presentation
}

\author{
Nyulas Tiberiu¹, Blendea Ciprian1,2, Rat Nora¹, Barcan Andreea1, Korodi Szilamer ${ }^{1}$, Bajka Balazs ${ }^{1,2}$ \\ 1 University of Medicine and Pharmacy of Tîrgu Mureș, Clinic of Cardiology, Tîrgu Mureș, Romania \\ 2 Cardio Med Medical Center, Department of Advanced Multimodal Imaging, Tîrgu Mureș, Romania
}

\begin{abstract}
Introduction: We present the case of a patient suffering from inferior vena cava hypoplasia complicated with Phlegmasia cerulean dolens. Imaging techniques allow precise diagnosis of inferior vena cava hypoplasia, providing essential structural details on the degree of damage of the vena cava and for the other branches. Case presentation: A 58 years old, obese and diabetic male patient presented with intense pain in the lower limbs, with the onset $24 \mathrm{~h}$ before presentation. The patient presented generalized edema, cyanosis and functional impotence. Angio CT examination revealed hypoplasia of the inferior vena cava, with extensive DVT (deep vein thrombosis). In emergency conditions, with the agreement of the patient, we initiated the thrombolytic therapy (streptokinase for $72 \mathrm{~h}$ ) associated with anticoagulants (heparin). The evolution was favorable: a significant reduction in leg circumference was recorded, together with pain relief and reduction of local inflammation in the lower limbs. Conclusion: Severe cases of inferior vena cava hypoplasia complicated with deep vein thrombosis can present a good prognosis if appropriate treatment with anticoagulants and thrombolytics is initiated in time.
\end{abstract}

Keyword: edema, CT scan, trombolysis, hypoplasia

Received: 25 September 2015 / Accepted: 07 November 2015

\section{Introduction}

Inferior Vena Cava (IVC) malformations such as absence of infrahepatic IVC or IVC hypoplasia are rare, being recorded in approximately $0.5 \%$ of the general population [1]. This condition is usually asymptomatic, the diseases being diagnosed incidentally, associated with other congenital anomalies such as situs inversus, congenital heart diseases, polysplenia and asplenia [2]. During physical exertion, the collaterals that are formed in the evolution of the disease are unable to withstand the increased blood flow, thereby generating venous stasis and clotting. Since during embryogenesis right metanephrons drain into the IVC, congenital absence of the latter could affect right kidney development. Some authors have suggested this condition as KILT syndrome (Kidney and IVC abnormalities with Leg thromboses) [3].

\section{Case report}

We present the case of a male patient, aged 58 years, who presented with intense pain in the lower limbs, with the onset $24 \mathrm{~h}$ before presentation. The patient presented generalized edema, cyanosis and functional impotence. From his personal history, we mentionthat the patient is suffering from essential hypertension stage II with high cardiovascular risk and non-insulin necessitating type II diabetes with renal and vascular complications. The physical examination revealed an influenced general condition, warm skin, pain, swelling, cyanosis of the lower limbs, generalized

* Correspondence to: Tiberiu Nyulas

E-mail: tiberiu.nyulas@gmail.com subdiafragmatic edema and bilateral basal crackles stasis.

Laboratory tests on admission were within normal limits. Urinalysis did not show any pathologic changes. ECG on admission showed no significant changes. The EcoDoppler examination of the lower limbs revealed a dilated inferior vena cava, with intraluminal hypoechogenic material, bilateral dilated external iliac veins, with intraluminal hypoechogenic content. The common femoral veins were dilated, with the lumen occupied with blood clots. The right femoral vein had a complete thrombosis on the whole length and an acute deep vein thrombosis completely occluding distal inferior vena cava was noted. Abdominal ultrasound revealed hepatic steatosis, chronic pancreatitis, hepatomegaly and kidney tumor formation on both kidneys. Also, at admission, an abdominopelvic AngioCT was performed, indicating hypoplasia of the inferior vena cava with azygos vein dilatation (fig. 1), perirenal venous dilatation (fig. 2), bilateral (on the right side pronounced) and infrarenal extensive DVT (of interest for the VCI, VIC internal and external, bilateral VFC); delayed bilateral renal function, bilateral renal cortical cysts; enlarged bilateral renal sinus (hydronephrosis). Concluding all clinical and paraclinical examinations, the patient was diagnosed with phlegmasia cerulean dolens and hypoplasia of the inferior vena cava.

Thrombolytic therapy was initiated (streptokinase for $72 \mathrm{~h}$ ), associated with anticoagulants (Heparin), with a significant amelioration in the clinical status. The circumference of the legthigh reduced and we also recorded a significant pain relief and reduction of inflammation signs on the lower limbs. After 8 days of treatment, AngioCT 


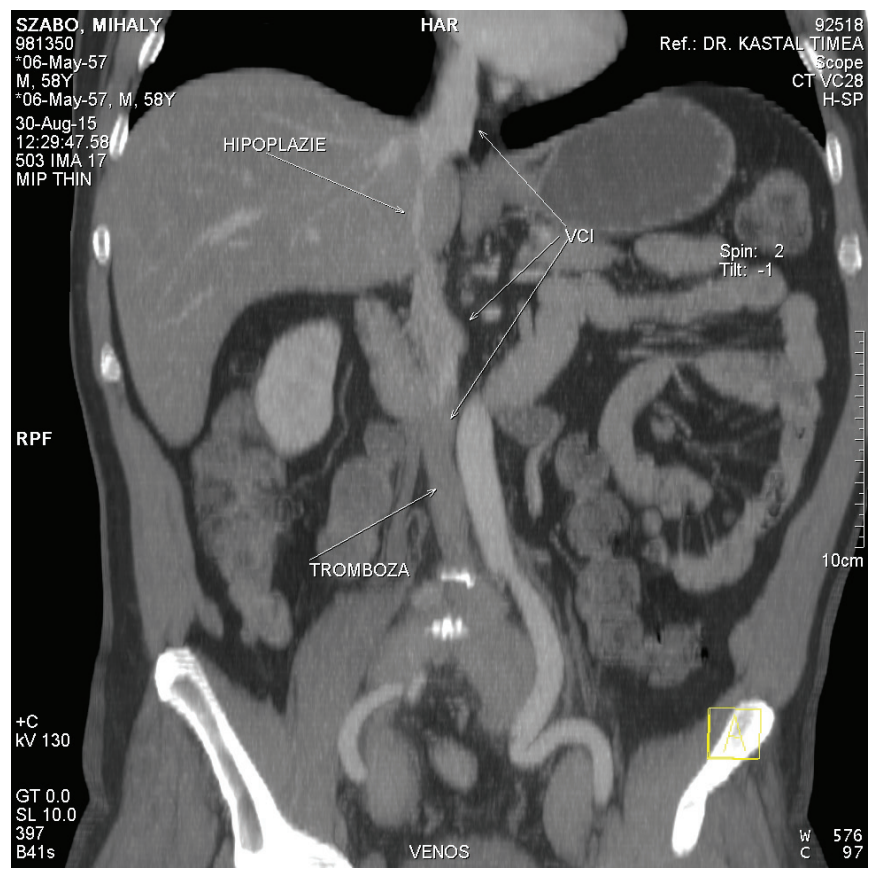

Fig. 1. Hypoplasia of the inferior vena cava

examination was repeated and found an arterial abdominal pelvic system and lower limb arterial system without detectable lesions (fig. 3), renal excretion present bilaterally, peri-testicular dilated varicose veins associating bilateral hydrocele (fig. 4) and deep venous system undergoing reperfusion with thrombotic debris at the level of inferior vena cava, common iliac vein confluence, left common iliac vein, bilateral internal iliac veins, right iliac vein, all associated with minimum peritoneal fluid collection.

\section{Discussions}

Patients with inferior vena cava hypoplasia are prone to develop deep vein thrombosis due to the venous stasis of

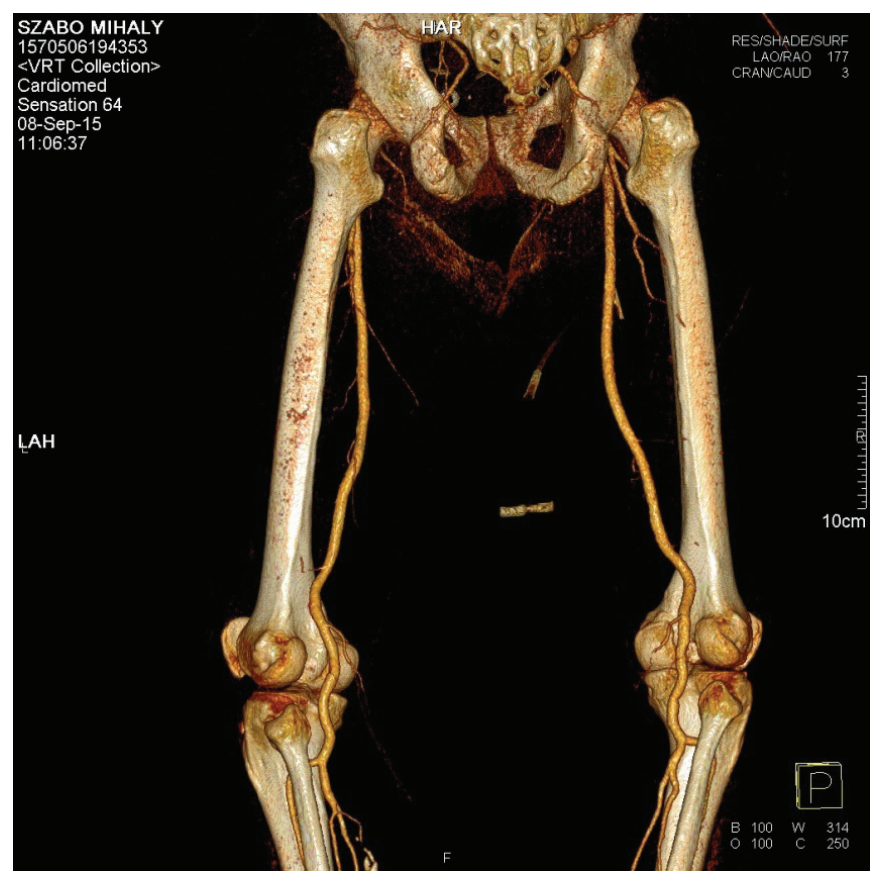

Fig. 3. Lower limb arterial system

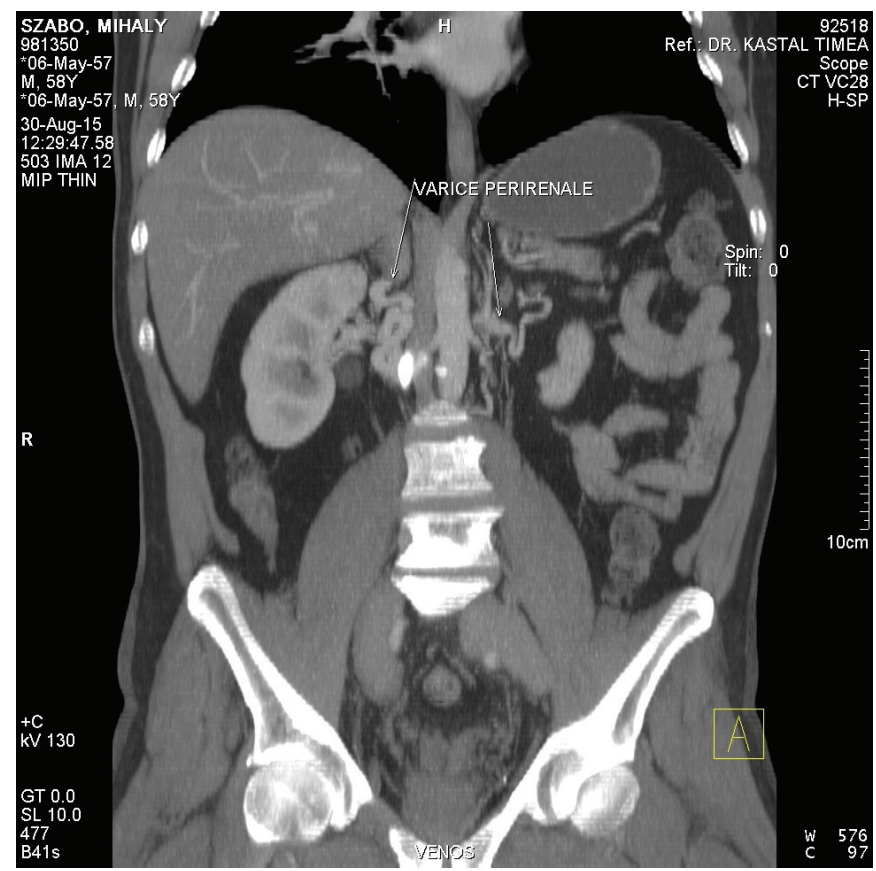

Fig. 2. Perirenal venous dilatation

the lower limbs [4]. In the case described, the patient had iliofemoral DVT of the lower limbs, which could be related to the hypoplasia of the vena cava inferior, revealed by imagistic evaluation. The presence of spontaneous, recurrent and sometimes bilateral proximal lower limb DVT must call the physician's attention to the possibility of an inferior vena cava anomaly [5]. MRI and contrast $\mathrm{CT}$ angiography are especially useful in such cases. However, DVT as a paraneoplasic manifestation or as a result of a hypercoagulable state should also be excluded. The most appropriate treatment in such cases is anticoagulation for at least six months. The possibility of recurrence is high when the anticoagulation treatment is discontin-

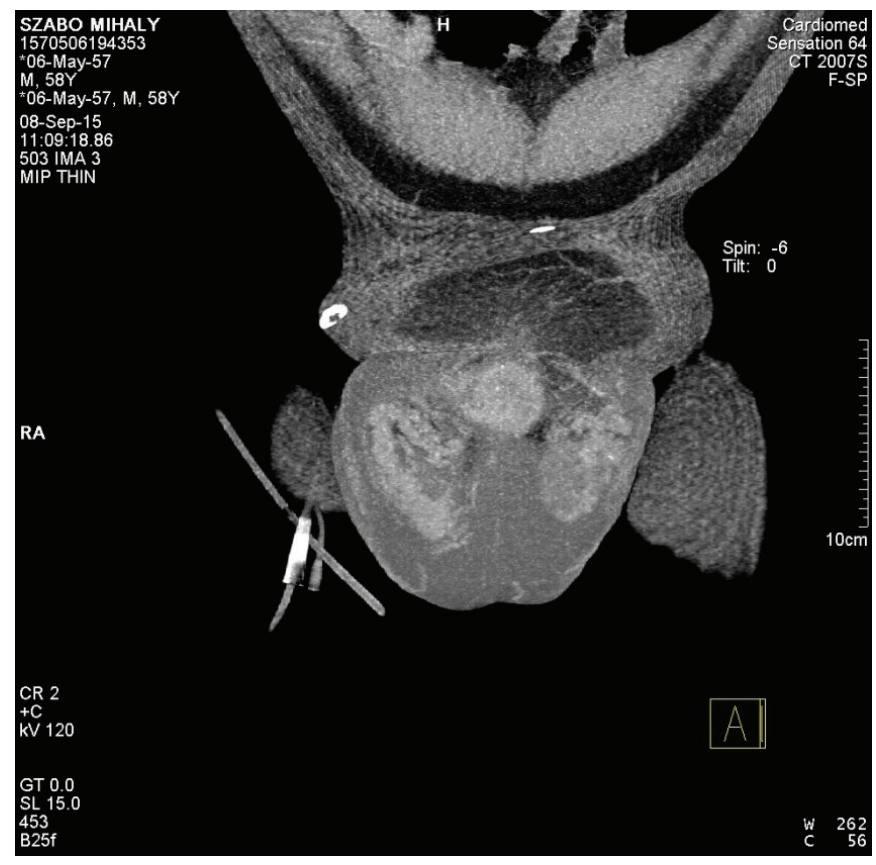

Fig. 4. Peritesticular dilated varicose veins 
ued before this period. Patients with IVC hypoplasia can present with different typesof clinical pictures. Some can be asymptomatic and hypoplasia of the IVC is found as an incidental finding, while others can present with venous thrombosis and its consequences as in our case.

\section{Conclusion}

Vena cava inferior atresia complicated with a deep vein thrombosis has a good prognosis under treatment with anticoagulants and thrombolytics.

\section{Acknowledgement}

This paper is supported by the Sectoral Operational Programme Human Resources Development (SOP HRD), financed from the European Social Fund and by the
Romanian Government under the contract number POSDRU/159/1.5/S/133377/

\section{References}

1. Sakellaris G, Tilemis S, Papakonstantinou O, et al. Deep venous thrombosis in a child associated with an abnormal inferior vena cava. ActaPaediatr. 2005;94:242-244.

2. Hamoud S, Nitecky S, Engel A, Goldsher D, Hayek T. Hypoplasia of the inferior vena cava with azygous continuation presenting as recurrent leg deep vein thrombosis. Am J Med Sci. 2000;319:414-416.

3. Gayer G, Zissin R, Strauss S, Hertz M. IVC anomalies and right renal aplasia detected on CT: A possible link? Abdom Imaging.2003;28:395-399.

4. Cho BC, Choi HJ, Kang SM, et al. Congenital absence of inferior vena cava as a rare cause of pulmonary thromboembolism. Yonsei Med J. 2004;45:947-951.

5. García-Fuster MJ, Forner MJ, Flor-Lorente B, et al. Inferior vena cava malformation and deep venous thrombosis. Rev EspCardiol. 2006;59:171-175. 